\title{
Integration of MALDI-TOFMS as high-throughput screening tool into the workflow of combinatorial polymer research
}

\section{Citation for published version (APA):}

Meier, M. A. R., \& Schubert, U. S. (2005). Integration of MALDI-TOFMS as high-throughput screening tool into the workflow of combinatorial polymer research. Review of Scientific Instruments, 76(6), 062211-1/6. https://doi.org/10.1063/1.1906123

DOI:

10.1063/1.1906123

Document status and date:

Published: 01/01/2005

\section{Document Version:}

Publisher's PDF, also known as Version of Record (includes final page, issue and volume numbers)

\section{Please check the document version of this publication:}

- A submitted manuscript is the version of the article upon submission and before peer-review. There can be important differences between the submitted version and the official published version of record. People interested in the research are advised to contact the author for the final version of the publication, or visit the $\mathrm{DOI}$ to the publisher's website.

- The final author version and the galley proof are versions of the publication after peer review.

- The final published version features the final layout of the paper including the volume, issue and page numbers.

Link to publication

\section{General rights}

Copyright and moral rights for the publications made accessible in the public portal are retained by the authors and/or other copyright owners and it is a condition of accessing publications that users recognise and abide by the legal requirements associated with these rights.

- Users may download and print one copy of any publication from the public portal for the purpose of private study or research.

- You may not further distribute the material or use it for any profit-making activity or commercial gain

- You may freely distribute the URL identifying the publication in the public portal.

If the publication is distributed under the terms of Article 25fa of the Dutch Copyright Act, indicated by the "Taverne" license above, please follow below link for the End User Agreement:

www.tue.nl/taverne

Take down policy

If you believe that this document breaches copyright please contact us at:

openaccess@tue.nl

providing details and we will investigate your claim. 


\title{
Integration of MALDI-TOFMS as high-throughput screening tool into the workflow of combinatorial polymer research
}

\author{
Michael A. R. Meier and Ulrich S. Schubert ${ }^{\mathrm{a})}$ \\ Laboratory of Macromolecular Chemistry and Nanoscience, Eindhoven University of Technology and Dutch \\ Polymer Institute (DPI), P.O. Box 513, 5600 MB Eindhoven, The Netherlands
}

(Received 29 October 2004; accepted 6 March 2005; published online 18 May 2005)

\begin{abstract}
The possibilities of an integration of matrix-assisted laser desorption/ionization time-of-flight mass spectrometry (MALDI-TOFMS) as an high-throughput screening tool into the workflow of combinatorial materials research are discussed. A multiple layer sample preparation technique for MALDI is described in detail and its possibilities of automation and miniaturization are discussed. Automated MALDI sample preparation could be performed within an automated synthesizer robot as well as with an ink-jet printer. The first approach offers the possibility of online reaction monitoring, whereas the second approach gives the opportunity of applications in ultra-high-throughput environments. Moreover, an example of high-throughput screening of a polymerization reaction by MALDI-TOFMS is discussed. () 2005 American Institute of Physics. [DOI: $10.1063 / 1.1906123$ ]
\end{abstract}

\section{INTRODUCTION}

Combinatorial materials research (CMR) can be considered as a rapidly growing and highly multidisciplinary field of research. ${ }^{1,2}$ One of its goals is the accelerated discovery and evaluation of new interesting materials by applying parallel and high-throughput approaches for the synthesis of these materials as well as for their property investigations. ${ }^{3-10}$ These recent successful examples clearly demonstrate that it is feasible to synthesize and investigate a large variety of different polymeric materials in a combinatorial fashion. However, CMR can only be successful if specially developed high-throughout screening (HTS) technologies for certain properties of interest are available. Examples of such tailor-made HTS techniques include, for instance the fast determination of molar mass and molar mass distribution by rapid gel permeation chromatography, ${ }^{11}$ the wettability of polymer libraries, ${ }^{12}$ or the morphology of polymer gradient libraries. ${ }^{13,14}$ However, until recently no feasible technique was described for the high-throughput determination of molar masses, molar mass distributions as well as of end-groups of synthetic polymers by mass spectrometry. Matrix-assisted laser desorption/ionization time-of-flight mass spectrometry (MALDI-TOFMS) has advanced to the perhaps most powerful MS tool for the analysis of polymers ${ }^{15}$ since its introduction in the 1980s. ${ }^{16,17}$ The oldest, simplest, and maybe most utilized sample preparation technique for MALDI-TOFMS is the dried-droplet (DD) method. ${ }^{15,18}$ For this method solutions of analyte, matrix, and ionizing salt (most likely in different solvents) are mixed by volume and an aliquot of the mixture (usually $0.5-1.0 \mu \mathrm{L}$ ) is deposited on the MALDI target and air dried. However, we recently developed a multiple-layer spotting technique for MALDI-TOFMS of synthetic polymers ${ }^{19}$ that is ideally suited for applications in combina-

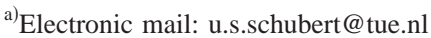

torial materials research. ${ }^{20,21}$ This method is able to significantly reduce the time required for sample preparation, ${ }^{22}$ to improve the analytical results ${ }^{19,23}$ as well as to be automated ${ }^{22,23}$ and miniaturized. ${ }^{23}$ Within this contribution we would like to provide an overview of this experimental measurement technique as well as to discuss benefits and limitations of it with regards to high-throughput screening in CMR.

\section{EXPERIMENT}

Matrices and inorganic salts were purchased from Sigma Aldrich (Oakville, Ontario, Canada). The investigated matrices were trans-2-[3-(4-tert-butylphenyl)-2-methyl-2-propenylidene]malononitrile (DCTB), $\alpha$-cyano-4-hydroxycinnamic acid (CAHA), 2,-(4-hydroxy-phenylazo)-benzoic acid (HABA), trans-3-indoleacrylic acid (IAA), 2,5dihydroxybenzoic acid (DHB), and 1,8,9-anthracenetriol (Dithranol). Analytical grade solvents were purchased from Biosolve LTD (Valkenswaard, The Netherlands). Poly(ethylene glycol) and poly(methyl methacrylate) standards were obtained from Polymer Standards Service GmbH (Mainz, Germany).

All MALDI experiments were carried out on VoyagerDE $^{\mathrm{TM}}$ PRO and STR Biospectrometry ${ }^{\mathrm{TM}}$ Workstations (Applied Biosystems, Foster City, CA, USA) time-of-flight mass spectrometers. All spectra were obtained in positive ion mode using linear mode for operation. Ionization was performed with a $337 \mathrm{~nm}$ pulsed nitrogen laser. All data were processed using the Data Explorer ${ }^{\mathrm{TM}}$ software package (Applied Biosystems, Foster City, CA, USA).

The automated spotting was carried out on a Chemspeed ASW2000 (Chemspeed Ltd., Augst, Switzerland) automated synthesizer. For the spotting a needle with $0.8 \mathrm{~mm}$ diameter was utilized in combination with a custom made MALDI target holder in a microtiter plate format (now commercially available from Chemspeed). 
(a)

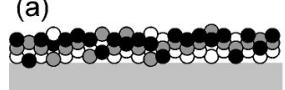

\section{(b)}

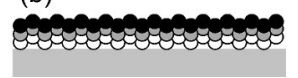

FIG. 1. Schematic representation of (a) conventional and (b) multiple-layer sample preparation for MALDI-TOFMS.

Ink-jet printing was carried out using an AutoDrop dropon-demand printer (Microdrop, Norderstedt, Germany). The setup consists of a MD-P-705-L xyz stage, on which a holder for the print head is mounted. An AD-K-501 micropipette was used as print head (nozzle diameter $70 \mu \mathrm{m}$ ).

\section{DEVELOPMENT OF A SUITABLE SAMPLE PREPARATION TECHNIQUE}

\section{A. General requirements}

The key factor and therefore the most important requirement for any successful MALDI experiment is an easily applicable and reproducible sample preparation technique. ${ }^{15}$ As already discussed in the Introduction, the most widely applied sample preparation for MALDI-TOFMS is the dried droplet method. One particular problem of this method for the analysis of synthetic polymers is the solvent selection, since all three compounds should be well soluble in a certain solvent. However, salts for instance, are, not well soluble in common organic solvents, which makes the DD method difficult for hydrophobic synthetic polymers. This can lead to a small amount of polymer nonsolvent in the final mixture, which can affect the signal reproducibility. ${ }^{18}$ Therefore, other sample preparation techniques for improved reproducibility and analytic results were developed including vacuum drying, ${ }^{24}$ overlayer (two-layer or seed layer), ${ }^{25,26}$ or fast evaporation. ${ }^{27}$ All mentioned methods have in common the effort to control the crystallinity of the utilized matrix and therefore the improvement of the analytical results. Concerning the high-throughput analysis of libraries by MALDITOFMS, especially the seed-layer technique could be auto- mated for the analysis of peptides and proteins ${ }^{26}$ as well as of a 41 compound library of organic molecules. ${ }^{28}$ In general, the requirements for an automated MALDI-TOFMS analysis of combinatorial libraries are similar to the above discussed ones. However, for combinatorial approaches a reproducible and widely applicable sample preparation technique is even more relevant, since a large variety of different compounds or, in the case of polymers, a large variety of different molecular weights has to be analyzed utilizing the same sample preparation technique. Moreover, the applied technique should be easy to automate, to integrate into the combinatorial workflow, and be as fast as possible. In order to meet these challenges a multiple-layer spotting technique was developed, evaluated, ${ }^{19}$ and later on automated and integrated into the workflow of combinatorial material research..$^{22,23}$

\section{B. Multiple layer spotting for MALDI-TOFMS}

The multiple layer spotting sample preparation technique for the MALDI-TOFMS of synthetic polymers was introduced recently and first evaluated with a series of poly(ethylene glycol) standards. ${ }^{19}$ Generally, it consists of a completely decoupled handling of the three main components of a MALDI-TOFMS sample, namely the matrix, the doping salt, and the analyte. These three components are spotted on top of one another in the mentioned order ${ }^{19}$ from different solutions, allowing the free choice of solvent for every component. As a result, difficulties in the solubility of analyte, salt, and matrix in different solvents can be avoided. Moreover, it was observed that this technique is able to provide superior analytical results if compared to the conventional dried droplet technique. Figure 1 depicts a schematic representation of the multiple layer spotting approach compared to a conventional dried droplet sample preparation technique. The main advantage of the multiple layer technique is the possibility to freely select a solvent/matrix combination that allows good crystallization of the matrix layer and therefore provides improved MALDI-TOFMS results. ${ }^{19,24,26,27}$ More-
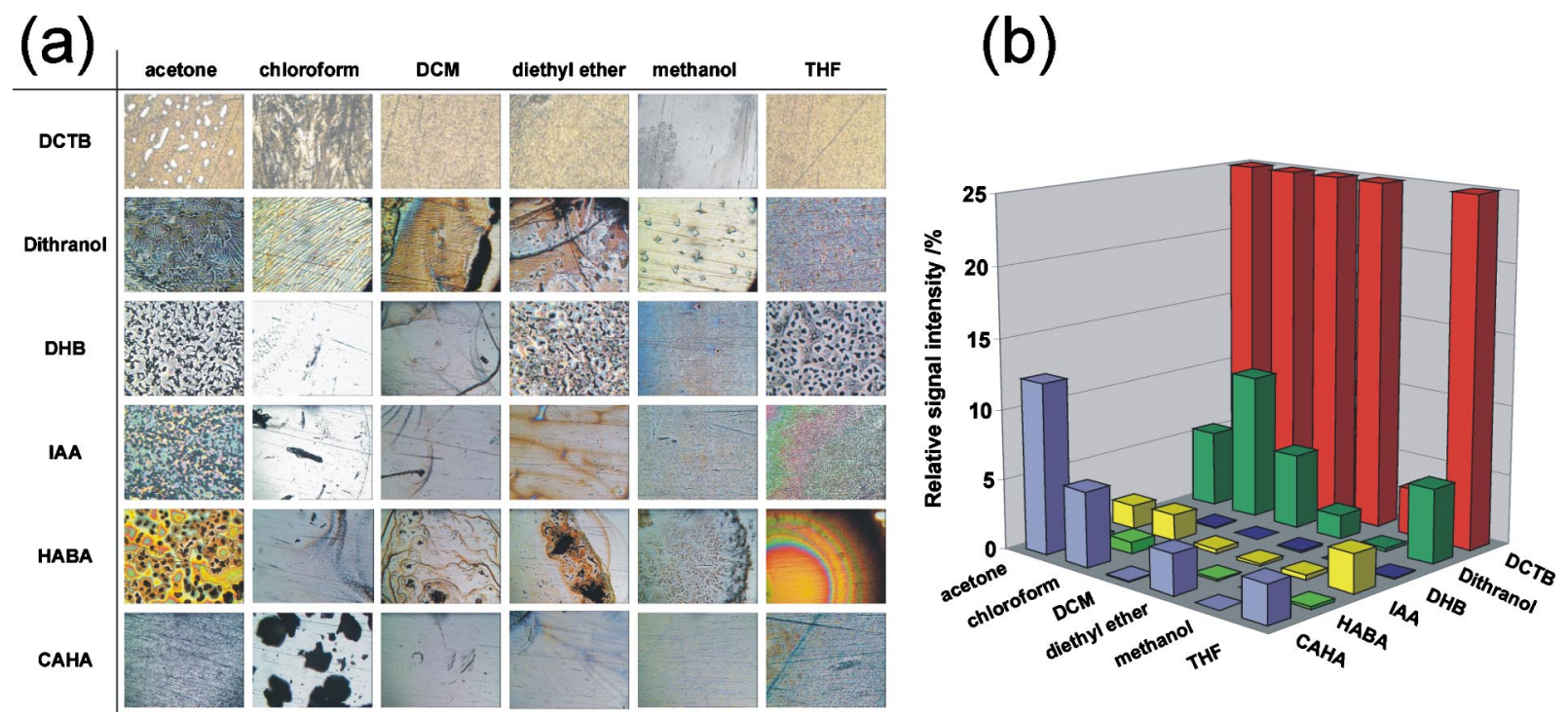

FIG. 2. (Color online) (a) Optical microscopy pictures of layers of different matrices crystallized from different solvents. (b) The corresponding MALDITOFMS signal intensities for a poly(ethylene glycol) standard $\left(M_{n}=5000 \mathrm{Da}\right)$. All MALDI spectra for this experiment were recorded with the same instrument settings for comparison reasons $(\mathrm{DCM}=$ dichloromethane, $\mathrm{THF}=$ tetrahydrofuran). 


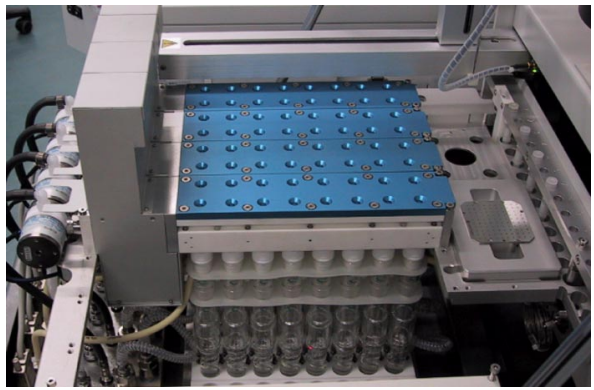

FIG. 3. (Color online) Inside view of the Chemspeed ASW 2000 automated synthesizer with reactor blocks installed and MALDI target in the specially designed holder.

over, a considerable time-saving effect was recognized when comparing the multiple layer spotting technique with the dried droplet technique, since the mixing step is completely removed from the protocol. Figure 2(a) shows optical microscopy pictures of the matrix layer of a 36 member library of MALDI-TOFMS samples that were prepared utilizing the multiple layer approach. Figure 2(b) displays the resulting relative signal intensities of this library when a sodium iodide in acetone and a poly(ethylene glycol) standard $\left(M_{n}\right.$ $=5000 \mathrm{Da})$ in dichloromethane were spotted on all 36 positions as second and third layers, respectively. For this MALDI-TOFMS experiment, spectra of all 36 samples were taken with the same instrument settings (e.g., laser intensity and delay time) and the corresponding signal intensities were subsequently compared. It was observed that DHB and HABA as matrices showed comparably low signal intensities, which is in good agreement with previously reported results. ${ }^{19}$ Moreover, the combination of dithranol as matrix with chloroform as solvent, which was the recently reported optimized result, provided the second highest signal intensity if the previously investigated matrices (dithranol, DHB, IAA, HABA, CHCA) are taken into account. However, the combination of CAHA as matrix and acetone as solvent provided even higher signal intensities. This optimized sample preparation was not identified in the previous evaluation since then a stepwise optimization was performed and not the whole library of samples was investigated in this study. However, the most striking result is that DCTB is the superior matrix and is not very selective on the solvent used for crystallization. All DCTB solvent combinations, except DCTB with methanol, were able to produce an at least tenfold higher signal intensity if compared to all other sample preparations. It should also be noted that the combination of DCTB and methanol (in contrast to the other DCTB solvent combinations) was not able to produce a crystalline surface as shown in Fig. 2(a), which resulted in the poor MALDI result for reasons already discussed above. Moreover, the trend that DCTB is able to produce considerably higher signal intensities if compared to other matrices is described in the literature for fullerene derivatives ${ }^{29}$ and was observed in our laboratories also for other classes of polymers. Therefore, DCTB in combination with the discussed multiple layer spotting approach for MALDI-TOFMS of synthetic polymers is the method of choice for high-throughput screening of synthetic polymers.

\section{INTEGRATION INTO THE WORKFLOW OF CMR}

\section{A. Automated synthesizer}

To automate the above described technique and to integrate MALDI-TOFMS monitoring possibilities into the workflow of CMR, the above described multiple layer sample preparation technique was integrated into an automated robotic synthesizer (Chemspeed ASW 2000) as described recently. ${ }^{22}$ Therefore, a custom made MALDI target holder in microtiter plate format was designed and the sample positions were programmed on an $x y z$ basis in the Chemspeed software (Gilson 735 Sampler Software V 2.10.). This software allows for programming the robot in a sequential fashion, including, for instance, the setting of temperature, reflux, vacuum, or gas supply. Moreover, it controls the liquid handling of the robotic arm in order to perform tasks such as the aspiration and dispensing of reactants from stock solutions into the reactor vessels or to take samples during reactions from the reactors. Figure 3 shows the MALDI target in the holder within the robotic synthesizer. The microtiter plate format of the holder allows an easy integration not only in the Chemspeed ASW 2000 robot, but as well into all other automated robotic systems available from Chemspeed and maybe other vendors, since the dimensions of microtiter plates are standardized. The actual sample preparation was performed utilizing the liquid handling system of the synthesizer robot. Therefore, $1 \mu \mathrm{L}$ aliquots of the three required components in different solutions (matrix, salt, and analyte) were spotted on top of one another. The time required to prepare a ready-to-measure sample with three layers of matrix, salt, and analyte in the given order is approximately 3 min. After this automated technique was evaluated and its
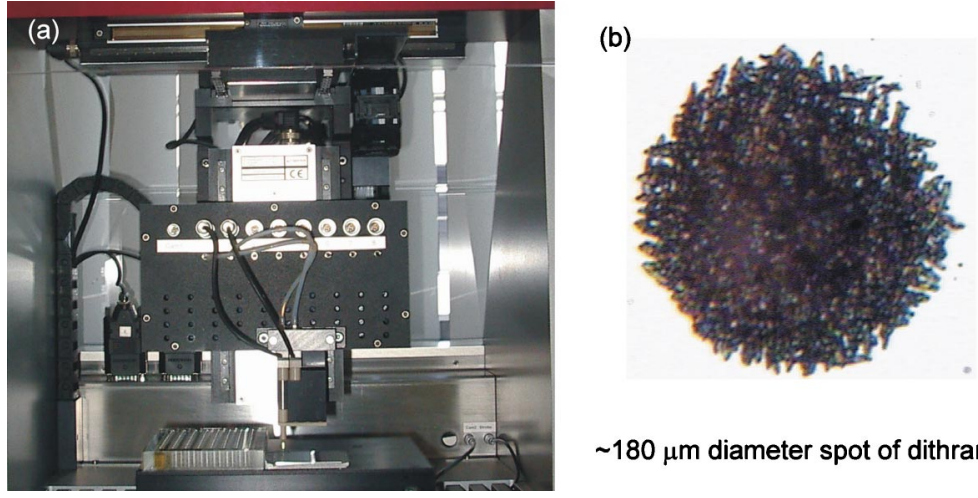

FIG. 4. (Color online) (a) Picture of the ink-jet printer with a microtiter plate containing the samples and the MALDI target. (b) A defined matrix spot resulting from the printing

$\sim 180 \mu \mathrm{m}$ diameter spot of dithranol 

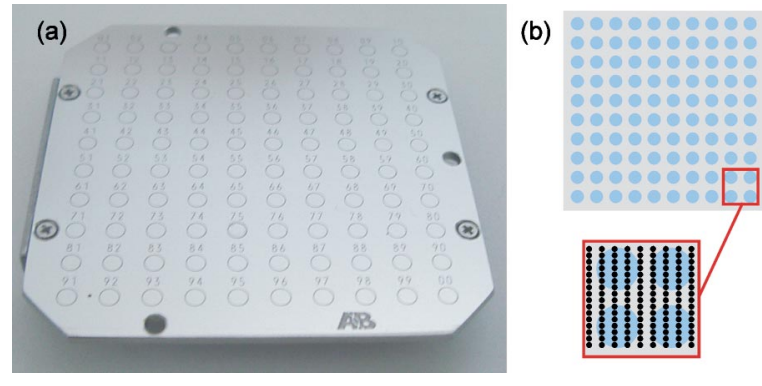

FIG. 5. (Color online) (a) Picture of a MALDI sample target. (b) Schematic representation of the target indicating the deposition of more than 4000 samples on one plate due to the small spot size of ink-jet printed samples.

reliability was confirmed with poly(styrene) standards of known molecular weight, it could be applied to the monitoring of the cationic ring opening polymerization of 2-ethyl-2-oxazoline. ${ }^{22}$ It was observed that the multiple layer approach was capable of reducing the time for sample preparation (approximately 90 seconds/sample) and maybe most importantly of offering improved analytical results. Moreover, valuable space within the automated synthesizer could be saved since no extra vials for the mixing of the three components are required.

\section{B. Ink-jet printing}

Ink-jet printing was utilized to further minimize and automate the multiple layer spotting technique for MALDI-TOFMS. ${ }^{23}$ Therefore, an ink-jet printer (compare Fig. 4) was used to deposit the three respective layers of matrix, salt, and analyte onto the MALDI target. Each layer consisted of five individual drops of the respective solution.
An array of $4 \times 4$ spots was considered as one MALDI sample. The sample preparation had to be optimized once more for poly(ethylene glycol) as well as for poly(methyl methacrylate) standards with regards to solvent/matrix combination, since the very volatile solvents applied in the previous approach were not compatible with the ink-jet printer. ${ }^{23}$ This resulted in very defined, homogenous, and small ( 180-200 $\mu \mathrm{m}$ diameter, compare Fig. 4) matrix layers if, for instance, dithranol was printed from an acetophenone solution. Therefore, also the analytical results were improved if compared to the dried droplet as well as to the conventional multiple layer spotting technique. Moreover, it was observed that MALDI spectra could be obtained from only a single spot and that the printing of an array $4 \times 4$ for MALDI measurements was not necessary. Therefore, this technique allows the deposition of more than 4000 MALDI samples on a conventional target (compare Fig. 5), which makes it suitable for applications of MALDI-TOFMS as a tool in ultra high-throughput applications. ${ }^{23}$

\section{SELECTED SCREENING RESULTS}

As an example for the application of the above described tools, the online monitoring of a reversible additionfragmentation chain transfer polymerization (RAFT) of methyl methacrylate (MMA) was chosen. For this monitoring approach, MALDI samples of poly(methyl methacrylate) (PMMA) were prepared with DCTB as matrix dissolved in tetrahydrofuran (THF) for reasons described above. The details of this investigation as well as all major results were already discussed recently. ${ }^{30}$ However, we would like to discuss some of the results in order to show that the above described combinatorial approaches can offer valuable ana-

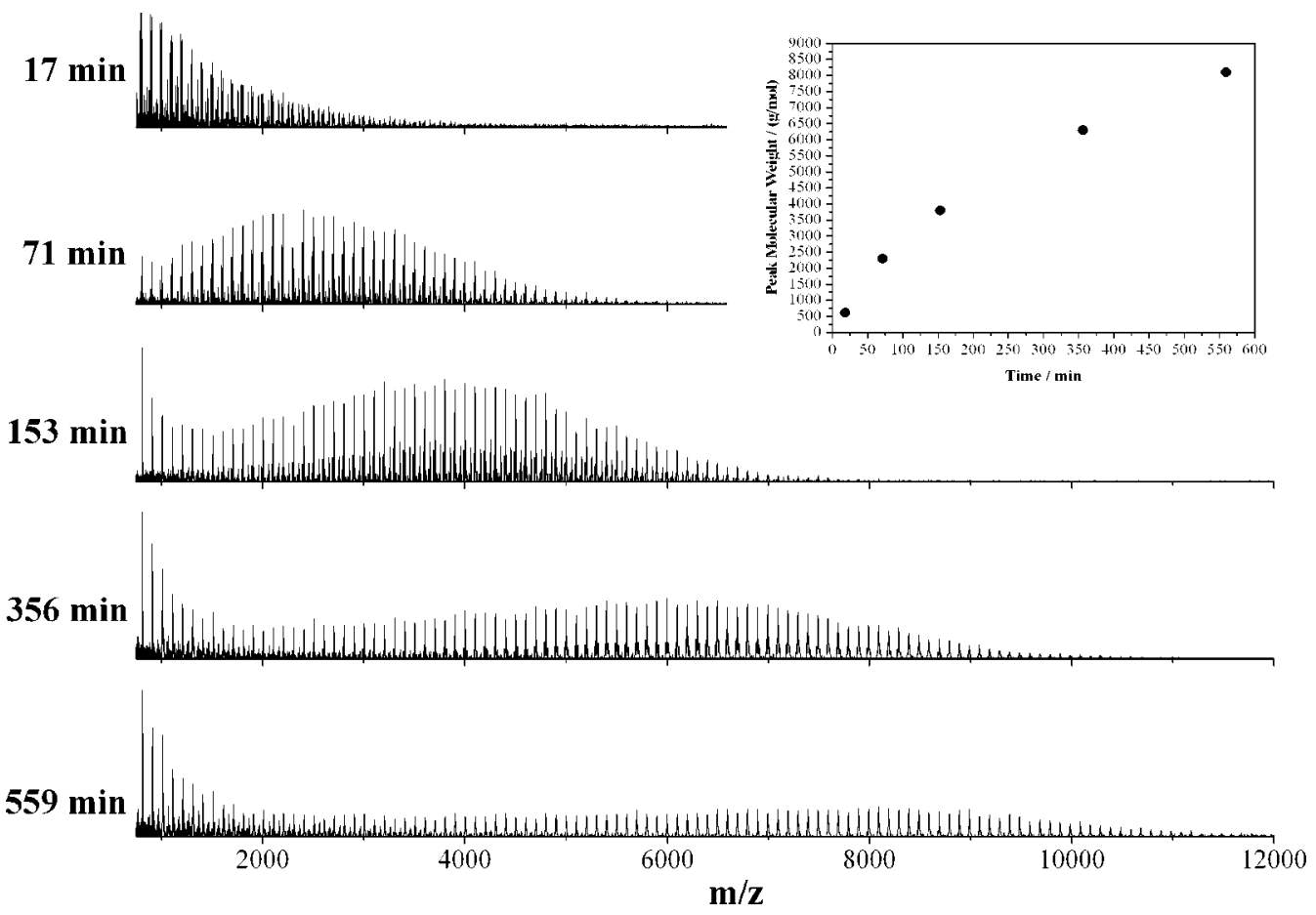

FIG. 6. MALDI-TOFMS monitoring of a RAFT polymerization in time: Spectra obtained from the automated spotting of the synthesized poly(methyl methacrylate) polymers at different reaction times. The insert shows the peak molecular weight $\left(M_{p}\right)$ (obtained from MALDI TOFMS spectra) as function of the reaction time (taken from Ref. 30). 
lytic information for an investigated polymerization system at an accelerated speed. RAFT polymerizations of methyl methacrylate (MMA) were performed on an Accelerator ${ }^{\mathrm{TM}}$ VLT100 automated synthesizer in order to investigate the controllability of this polymerization under different conditions. Therefore, different RAFT-agent to initiator concentrations were investigated with azoisobutyronitrile as initiator at $70{ }^{\circ} \mathrm{C}$ and a polymerization time of $9.5 \mathrm{~h}$. Thereby, each RAFT-agent to initiator ratio reaction was performed four times to investigate the reproducibility. During these automated polymerizations, samples were taken for automated GPC as well as MALDI investigations. Automated MALDITOFMS as well as GPC analysis revealed that reactions that were performed under the same conditions showed similar molecular weights and molecular weight distributions, providing first proofs of the reproducibility of these reactions. Monitoring reactions in time by MALDI-TOFMS (compare Fig. 6) revealed a curved increase of the molecular weight in time, as it can be expected for a controlled polymerization technique, since a decreasing monomer concentration in time in combination with the constant radical concentration during the polymerization results in this behavior. Peak molecular weights were utilized for the visualization of the polymer chain growth in time (compare inset, Fig. 6). These results were highly comparable for all four reactions performed under equal conditions. Further MALDI investigations were able to visualize automated chain extension experiments and allowed end-group determinations of the investigated polymers giving further proof of the controllability of the automatically performed RAFT polymerizations. In general, the results obtained from this MALDI monitoring experiments were highly comparable and provided additional proofs for the controllability and reproducibility of the investigated polymerization system. This example of high-throughput screening with MALDI-TOFMS nicely demonstrates that automated MALDI-TOFMS is a valuable tool for the investigation of polymerization kinetics and that screening results are not necessarily of bad quality.

\section{ACKNOWLEDGMENTS}

This study was supported by the Dutch Polymer Institute (DPI) and the Fonds der Chemischen Industrie. The authors thank the co-authors of the individual scientific papers that were the basis of this overview for their help and support.

${ }^{1}$ Special Issue, Macromol. Rapid Commun. 24, 3 (2003).

${ }^{2}$ Special Issue, Macromol. Rapid Commun. 25, 19 (2004).

${ }^{3}$ S. Brocchini, K. James, V. Tangpasuthadol, and J. Kohn, J. Am. Chem. Soc. 119, 4553 (1997).

${ }^{4}$ D. M. Lynn, D. G. Anderson, D. Putnam, and R. Langer, J. Am. Chem. Soc. 123, 8155 (2001).

${ }^{5}$ H. Zhang, M. W. M. Fijten, R. Reinierkens, R. Hoogenboom, and U. S. Schubert, Macromol. Rapid Commun. 24, 81 (2003).

${ }^{6}$ M. A. R. Meier, J.-F. Gohy, C.-A. Fustin, and U. S. Schubert, J. Am. Chem. Soc. 126, 11517 (2004).

${ }^{7}$ R. Hoogenboom and U. S. Schubert, J. Polym. Sci., Part A: Polym. Chem. 41, 2425 (2003).

${ }^{8}$ O. Lavastre, I. Illitchev, G. Jegou, and P. H. Dixneuf, J. Am. Chem. Soc. 124, 5278 (2002).

${ }^{9}$ J. C. Carnahan, J. P. Lemmon, T. K. Leib, R. A. Potyrailo, and G. L. Warner, (General Electric Company) U.S. Patent No. 6,307,004 (2001).

${ }^{10}$ R. A. Potyrailo, R. J. Wroczynski, J. P. Lemmon, W. P. Flanagan, and O. P. Siclovan, J. Comb. Chem. 5, 8 (2003).

${ }^{11}$ H. Pasch and P. Kilz, Macromol. Rapid Commun. 24, 104 (2003).

${ }^{12}$ J.-F. Thaburet, H. Mizomoto, and M. Bradley, Macromol. Rapid Commun. 25, 366 (2004).

${ }^{13}$ J. C. Meredith, A. P Smith, A. Karim, and E. J. Amis, Macromolecules 33, 9747 (2000).

${ }^{14}$ A. P. Smith, J. F. Douglas, J. C. Mederith, E. J. Amis, and A. Karim, J. Polym. Sci., Part B: Polym. Phys. 39, 2141 (2001).

${ }^{15}$ S. D. Hanton, Chem. Rev. (Washington, D.C.) 28, 4562 (2001).

${ }^{16}$ K. Takana, H. Waki, Y. Ido, S. Akita, Y. Yoshida, and T. Yoshida, Rapid Commun. Mass Spectrom. 2, 151 (1988).

${ }^{17}$ M. Karas and F. Hillenkamp, Anal. Chem. 60, 2299 (1988).

${ }^{18}$ M. W. F. Nielen, Mass Spectrom. Rev. 18, 309 (1999).

${ }^{19}$ M. A. R. Meier and U. S. Schubert, Rapid Commun. Mass Spectrom. 17, 713 (2003).

${ }^{20}$ M. A. R. Meier and U. S. Schubert, J. Mater. Chem. 14, 3289 (2004).

${ }^{21}$ M. A. R. Meier, R. Hoogenboom, and U. S. Schubert, Macromol. Rapid Commun. 25, 21 (2004).

${ }^{22}$ M. A. R. Meier, R. Hoogenboom, M. W. M. Fijten, M. Schneider, and U. S. Schubert, J. Comb. Chem. 5, 369 (2003).

${ }^{23}$ M. A. R. Meier, B. J. de Gans, A. M. J. van den Berg, and U. S. Schubert, Rapid Commun. Mass Spectrom. 17, 2349 (2003).

${ }^{24}$ D. I. Papac, A. Wong, and A. J. Jones, Anal. Chem. 68, 3215 (1996).

${ }^{25}$ Y. Dai, R. Whittal, and Liang Li, Anal. Chem. 71, 1087 (1999).

${ }^{26}$ P. Önnerfjord, S. Ekström, J. Bergquist, J. Nilsson, T. Laurell, and G. Marko-Varga, Rapid Commun. Mass Spectrom. 13, 315 (1999).

${ }^{27}$ O. Vorm, P. Roepstorff, and M. Mann, Anal. Chem. 66, 3281 (1994).

${ }^{28}$ D. A. Lake, M. V. Johnson, C. N. McEwen, and B. S. Larsen, Rapid Commun. Mass Spectrom. 14, 1008 (2000).

${ }^{29}$ L. Ulmer, H. G. Torres-Garcia, J. Mattay, and H. Luftmann, Eur. J. Mass Spectrom. 6, 49 (2000).

${ }^{30}$ M. W. M. Fijten, M. A. R. Meier, R. Hoogenboom, and U. S. Schubert, J. Polym. Sci., Part A: Polym. Chem. 42, 5775 (2004). 